\title{
Evolution as Fact, Theory, and Path
}

\author{
T. Ryan Gregory
}

Published online: 20 November 2007

(C) Springer Science + Business Media, LLC 2007

Keywords Terminology · Science · Language · Vernacular . Hypothesis · Fact · Theory $\cdot$ Evolution · Darwin

\section{Introduction}

With its vocabulary of hundreds of thousands of words, one might expect English to boast a surplus of ways to express different concepts. Indeed, there are many well-known examples of multiple descriptors for the same item or idea, often one or more from the Germanic and others from the Latinate roots of modern English. In addition to the diversity resulting from a history of linguistic hybridization, English has a tendency to assimilate words from other languages and to include the de novo creation of terms as the need arises. Thus, most technically complex professions exhibit a plethora of neologisms and jargon that can be all but impenetrable to nonexperts. Science is certainly no exception in this regard.

However, when it comes to some of the most fundamental concepts in science, there is a dearth of unambiguous terminology. Worse still, words with relatively clear meanings in the vernacular are employed with very different definitions in science, a phenomenon that greatly confuses discussions of science when they are conducted in nonscientific contexts. For example, terms such as "energy" or "force" have specific meanings in physics that are easily confused when commingled with their common usages. This ambiguity has been exploited to considerable advan-

T. R. Gregory $(\bowtie)$

Department of Integrative Biology, University of Guelph,

Guelph, ON N1G 2W1, Canada

e-mail: rgregory@uoguelph.ca tage by many a huckster who falsely invokes the respectability of science in the sale of products that would, in actuality, contradict well established scientific principles if they really exerted any of their claimed effects.

Even more generally, terms relating to the process and products of science itself, such as "theory" and "law", are almost diametrically opposite in scientific vs vernacular settings. This has been a source of both honest confusion and intentional obfuscation in discussions of science, especially with regard to evolution-which has, with the full thrust of equivocation, been misleadingly labeled as "just a theory" by opponents for decades. The intent of this article is to clarify the general meaning of some central concepts in science and the terms used to describe them, and to differentiate these from the very different definitions of the same words in common usage. The specific application of these terms, as defined in science, to the topic of evolution will be discussed in some detail.

\section{Defining Terms}

Hypothesis, theory, fact, law. Prefaced with "hunch" or "guess", this list of terms would reflect what many people consider a graded series from least to greatest degree of certainty. This ranking may be appropriate in common usage, but actually makes little sense when these words are employed in a scientific context.

\section{Fact}

"Fact" is perhaps the only term in the above list whose common and technical definitions are similar. The major difference is in the degree of certainty expressed, which is 
simultaneously higher and lower in scientific usage. Following the definition provided by the US National Academy of Science (NAS) (1998), one of the most prestigious scientific societies in the world, a scientific fact is "an observation that has been repeatedly confirmed, and for all practical purposes, is accepted as 'true'." Or, as Stephen Jay Gould (1981) put it in his inimitable style, "In science, 'fact' can only mean 'confirmed to such a degree that it would be perverse to withhold provisional assent'." It is this insistence on repeated confirmation by data - either through direct observation or reliable inference-that makes a claim to "fact" so much stronger in science. However, as the NAS points out, "truth in science is never final, and what is accepted as a fact today may be modified or even discarded tomorrow". Small-scale details are regularly revised as more precise observations are made, whereas well established facts of fundamental significance are very rarely overthrown, but in principle, no scientific fact of any magnitude is beyond revision or refutation. As a result, scientists must maintain a balance between the confidence that comes from reinforcing conclusions about the world with repeatable data and the understanding that absolute certainty is not something that the methods of science are able or intended to deliver.

\section{Theory}

The common and scientific definitions of "theory," unlike of "fact," are drastically different. In daily conversation, "theory" often implicitly indicates a lack of supporting data. Indeed, introducing a statement with "My theory is..." is usually akin to saying "I guess that...", "I would speculate that...", or "I believe but have not attempted to demonstrate that...". By contrast, a theory in science, again following the definition given by the NAS, is "a well-substantiated explanation of some aspect of the natural world that can incorporate facts, laws, inferences, and tested hypotheses." Science not only generates facts but seeks to explain them, and the interlocking and well-supported explanations for those facts are known as theories. Theories allow aspects of the natural world not only to be described, but to be understood. Far from being unsubstantiated speculations, theories are the ultimate goal of science.

\section{Hypothesis}

The validity of scientific theories is not determined solely by their ability to accommodate and account for known facts. Theories also are actively tested, and it is here that "hypotheses" play an important role. According to the NAS, a scientific hypothesis is "a tentative statement about the natural world leading to deductions that can be tested." Testing can involve direct experimentation or the genera- tion of predictions about as-yet-unobserved facts that can be evaluated by further observation. This latter process plays a significant part in the validation of theories in sciences such as astronomy and geology where direct experimental manipulation is difficult. As the NAS notes, "If the deductions are verified, the hypothesis is provisionally corroborated. If the deductions are incorrect, the original hypothesis is proved false and must be abandoned or modified." It bears noting that the rejection of a hypothesis does not automatically imply the refutation of an entire theory because hypotheses are usually sufficiently focused to test only one aspect of complex theories.

\section{Law}

Finally, "law", for which, once again, there is a nearly opposite definition in everyday use compared to the application of the term in science. "Laws," in normal experience, are prescriptive - that is, they dictate what behaviors one should carry out and which ones must be avoided. A posted speed limit, for example, (attempts to) dictate the behaviors of drivers. A scientific law, on the other hand, is descriptive - it is a "generalization about how some aspect of the natural world behaves under stated circumstances" according to the NAS. In the vernacular, a law prescribes behavior and limits what is permitted to happen. In science, a law describes and predicts what will happen when the range of possible conditions is limited. If one is caught speeding, then mechanisms are implemented to correct this deviation from externally imposed limits. However, there is no punishment for "violating the laws of physics" or "defying the law of gravity" because these phrases are nonsensical from a scientific standpoint.

More specifically, if an observation does not conform to the expectations of a scientific law, then either (1) the observation was illusory or interpreted incorrectly, (2) the observed event took place outside the specified conditions to which the law applies, or (far less likely), (3) the law is inaccurately formulated. A prime example is provided by the chronically misunderstood Second Law of Thermodynamics, which states that "the entropy of a closed system not in equilibrium will tend to increase over time, approaching a maximum value at equilibrium." In this case, the conditions are very clearly specified: if there is no external source of energy ("a closed system"), then there will be a net increase in disorder until the system reaches equilibrium. Local increases in order are not precluded (ornate snowflakes still form from water vapor), and of course, this does not apply to living things, which draw energy from their environments (and ultimately from the sun), and hence, represent open systems. Readers of this article establish this latter claim conclusively, having passed from a simple zygote to a complex organism composed of 
trillions of specialized cells. If the Second Law of Thermodynamics implied that all natural increases in order were impossible, then it would be incorrect. It does not and (so far as we know) is not. The broader point is that invoking the Second Law of Thermodynamics as an argument against evolution reveals a misunderstanding of both the scope of this particular law and of the meaning of "law" in science generally.

Theories explain facts and are tested by generating hypotheses. No matter how much information accrues, hypotheses never become theories, and theories never graduate into laws. These terms describe three distinct aspects of science.

\section{An Attractive Case Study}

The scientific application of words that are used in very different ways under normal circumstances can appear rather counterintuitive, to be sure. In this sense, it is instructive to consider a case study with which all readers of this article are at least casually familiar: gravity. Some facts-observations repeatedly confirmed and considered accurate-about gravity include the following:

(1) Even though the Earth is rotating rapidly, physical objects on its surface (say, readers of this article) are not flung off into space.

(2) If one drops something or throws it into the air, it falls to the ground.

(3) Objects dropped in the same location accelerate under gravity at the same rate, regardless of their mass (wind resistance notwithstanding).

The first of these is being observed and confirmed at this very moment all over the planet. The second can be tested at any time (feel free to confirm it using a nonbreakable object of your choosing). The third is far less intuitive, and in fact, required the genius of Galileo to demonstrate as a scientific principle and subsequent researchers with more sophisticated instruments to confirm. The equal rates of acceleration of objects independent of mass can be demonstrated by using objects of similar shape (to cancel out differences in friction with the air-try, say, a baseball and a basketball; Galileo used balls rolling down inclines), and this can be observed using any objects in an artificially created vacuum. However, one of the most dramatic demonstrations came when Dave Scott, an astronaut on the Apollo 15 mission to the airless surface of the moon, dropped a hammer and a feather and observed the result. "How about that," he noted, "Mr. Galileo was correct".

Gravity cannot be observed directly, such that its characteristics must be inferred from observations of its effects. These effects, it turns out, can be predicted with extreme accuracy if specific conditions are identified-in other words, there are laws that can describe the behavior of objects under the influence of gravity. First, the force exerted by gravitation can be described as the product of an object's mass multiplied by its acceleration. This is known as Newton's Second Law of Motion. Moreover, Newton's Law of Universal Gravitation specifies that the force of gravity experienced by two objects is proportional to the product of their masses and inversely proportional to the square of the distance between them. In other words, if one specifies the masses of two objects and their distance from one another, one can calculate with great precision the force of gravity to which they will be subject and how they will behave as a consequence. Deviations from the expected orbit of Uranus based on physical laws allowed the existence and location of another massive object to be predicted - the object in question, the planet Neptune, was discovered in 1846 within $1^{\circ}$ of its inferred position.

Of course, acknowledging, describing, or even predicting the effects of gravity do not explain how the phenomenon works or why it has the properties that it does. Facts and laws are insufficient for a deeper understanding of gravitation; to achieve this requires a testable, substantiated, comprehensive explanation that is consistent with all known facts about gravity - in other words, a theory. Many theories of gravitation have been proposed, and most of them have failed tests or have been inconsistent with accepted facts and have therefore been rejected. The reigning theory, Einstein's Theory of General Relativity, explains gravity as the consequence of the warping of space-time, the fabric of the universe, by mass. Einstein's theory has triumphed to date because it has been able to account for observations that other theories (e.g., Newton's) could not, such as the characteristics of the orbit of Mercury and the bending of light by mass. In fact, it was a test of the latter, during an eclipse in 1919, that made Einstein an international celebrity.

Relativity, like any other theory in science, continues to be tested. Notably, Kramer et al. (2006) recently highlighted the utility of a double pulsar system in space as "a good candidate for testing Einstein's theory of general relativity and alternative theories of gravity". The fact that global positioning satellite (GPS) systems would not work without correcting for the implications of relativity represents an indirect confirmation as well.

Einstein's theory is not complete, however. For one, it has not been possible thus far to reconcile relativity with observations (rather, inferences) about the nature of the universe at the subatomic scales at which quantum processes operate. It also does not explain why gravity is such a weak force relative to the other known forces (electromagnetism and the strong and weak nuclear forces). Indeed, a trivial application of electromagnetic force is 
more than sufficient to counteract the force of gravity exerted by the entire planet, as when a paperclip is lifted with a small magnet. Explaining these properties of gravity remains an active area of research in theoretical physics (Randall 2005).

\section{Evolution as Fact}

The notion that species may change through time and that living organisms are related to one another through common descent was not original to Charles Darwin. Ideas regarding evolutionary change, as with ideas about gravity, extend back at least to a few ancient Greek thinkers. There had been much discussion of this topic two generations before Darwin based on the writings of Jean-Baptiste de Lamarck, and Darwin's own grandfather, Erasmus Darwin, was explicit in his view that species could change. Darwin's major contribution on this issue was not to introduce the idea, but to assemble a massive compendium of data in support of what he called "descent with modification".

In The Origin of Species, published in 1859, Darwin cited independent lines of evidence such as the biogeographical distribution of species, homology of structure, the occurrence of vestigial organs and atavisms, and the already well established process of extinction as all pointing to a conclusion that species have changed over time and are connected by descent from common ancestors. Through the force of Darwin's argument and the mass of supporting data he presented, it was not long before the contemporary scientific community came to acknowledge the historical reality of evolutionary descent. As A.W. Bennett summarized the situation in 1870 ,

The fascinating hypothesis of [descent with modification] has, within the last few years, so completely taken hold of the scientific mind, both in [Great Britain] and in Germany, that almost the whole of our rising men of science may be classed as belonging to this school of thought. Probably since the time of Newton no man has had so great an influence over the development of scientific thought as Mr. Darwin.

Over the past 150 years, this initial list has been supplemented by countless observations in paleontology, comparative anatomy, developmental biology, molecular biology, and (most recently) comparative genomics, and through direct observations of evolutionary change in both natural and experimental populations. Each of thousands of peer-reviewed articles published every year in scientific journals provides further confirmation (though, as Futuyma (1998) notes, "no biologist today would think of publishing a paper on 'new evidence for evolution' ... it simply hasn't been an issue in scientific circles for more than a century"). Conversely, no reliable observation has ever been found to contradict the general notion of common descent. It should come as no surprise, then, that the scientific community at large has accepted evolutionary descent as a historical reality since Darwin's time and considers it among the most reliably established and fundamentally important facts in all of science.

\section{Evolution as Theory}

Establishing the fact of evolution was only half of Darwin's objective. He also sought to explain this fact by proposing a mechanism: his Theory of Evolution by Natural Selection. As he stated in 1871, "I had two distinct objects in view; firstly, to show that species had not been separately created, and secondly, that natural selection had been the chief agent of change."

Natural selection was neither the first nor the last theory proposed to explain the fact of evolution. Lamarck's theory, in particular, had been based on two key ideas: "use and disuse" and the inheritance of acquired characteristics. In combination, these suggested that the traits acquired through use by organisms during their lifetimes would be passed on to offspring (for example, that the children of individuals who exercise vigorously would be born with greater musculature) and conversely that features that went unused would be lost. (The notion that evolutionary change occurs in response to need in combination with an internal striving toward greater perfection is a common misconception that is often attributed to Lamarck; see Kampourakis and Zogza 2007 for criticism of this practice). Lamarck's proposed mechanism is not compatible with the modern understanding of genetics and has therefore been abandoned. However, it seems that it and the notion of striving to fulfill needs are more intuitive than Darwinian natural selection, ${ }^{1}$ which probably explains why they were proposed first and why so many students and others continue to conceive of evolution in these inaccurate terms (Bishop and Anderson

\footnotetext{
${ }^{1}$ The components of evolutionary biology dealing specifically with natural selection are "Darwinian" (as opposed to, say, "Lamarckian" mechanisms). Similarly, descriptions of gravity can legitimately be considered "Newtonian" or "Einsteinian" depending on which theory is being invoked. By contrast, the labels "Darwinism" for an acceptance of the fact of evolution and "Darwinists" for those who acknowledge common descent as a historical reality are used primarily as a pejorative description by antievolutionists. It is roughly equivalent to using a moniker such as "Newtonism" to describe the acceptance of the physical reality of gravity or "Einsteinists" for those who acknowledge gravity to be a fact. Scientists who study evolution are properly known as "evolutionary biologists" or sometimes "evolutionists".
} 
1990; Demastes et al. 1995; Alters and Nelson 2002). A similar phenomenon has been observed in physics education, in which Newtonian or Einsteinian ideas taught to students must compete with incorrect but evidently more intuitive Aristotelian preconceptions (Halloun and Hestenes 1985a,b).

Although he succeeded in establishing the fact of evolution in short order, Darwin did not live to see natural selection adopted as a central mechanism in evolutionary theory. In fact, by the dawn of the 20th century, natural selection had been nearly eclipsed as a favored mechanism of evolutionary change. Theories involving instantaneous, rather than gradual, changes (mutationism), internal forces creating a sort of unavoidable evolutionary inertia even to the point of extinction (orthogenesis), and renewed appeals to use and disuse (neo-Lamarckism) had moved into the spotlight (Bowler 1992). It was not until the "Modern Synthesis" of the 1930s and 1940s that natural selection returned to the fore when it was shown to be compatible with Mendel's laws of inheritance ${ }^{2}$ (Mayr and Provine 1980; Bowler 2003). Darwin himself had no knowledge of genetics, making this revised version sufficiently distinct from the original to qualify as "neo-Darwinian theory".

Modern evolutionary theory represents a multifaceted set of explanations for patterns observed both in contemporary populations and in deep time as revealed by the fossil record. Natural selection is considered by many to be the prime component of evolutionary theory and is the only workable mechanism ever proposed that is capable of accounting for the adaptive features of organisms. At the molecular level, nonadaptive mechanisms are recognized as highly significant, and there is also an increasing emphasis on changes due to processes such as genetic drift that differ from natural selection by being due to chance. ${ }^{3}$

Because of this complexity, biologists rarely make reference to "the theory of evolution," referring instead simply to "evolution" (i.e., the fact of descent with modification) or "evolutionary theory" (i.e., the increasingly

\footnotetext{
${ }^{2}$ Being scientific laws, these describe the outcome of a system under defined conditions, specifically, the way that genes are inherited by offspring in organisms with sexual reproduction and two copies of the chromosome set (diploids), in the absence of any complicating factors.

${ }^{3}$ Genetic drift involves changes in the genetic composition of populations due to chance events and is most powerful in small populations. It is also widely recognized that mutations, which are the source of the genetic variation upon which other evolutionary processes depend, is "random" in the sense that mutations occur without regard to their consequences for organisms, although not all are equally likely. Natural selection, on the other hand, is the opposite of random chance. While there are chance elements involved (mutation and genetic drift), it is a deep misconception to equate evolution as a whole to random chance.
}

sophisticated body of explanations for the fact of evolution). That evolution is a theory in the proper scientific sense means that there is both a fact of evolution to be explained and a well-supported mechanistic framework to account for it. To claim that evolution is "just a theory" is to reveal both a profound ignorance of modern biological knowledge and a deep misunderstanding of the basic nature of science.

\section{Evolution as Path}

Some scientific disciplines-geology, archeology, astronomy, and evolutionary biology among them-deal not only with general processes and mechanisms, but also unique historical particulars. In addition to its incarnations as a "fact" and a "theory," evolution also can be discussed in a third distinct capacity, namely, as a "path" (Ruse 1997). Evolution as path deals with the factual details of life's history, such as the degree of relatedness of modern species to one another, the timing of splits among lineages, the characteristics of extinct ancestors, and the major events that have occurred over the nearly 4 billion years of life's saga. As an example, specialists including paleontologists and molecular systematists may investigate whether birds are the descendants of a lineage of dinosaurs (and if so, which one), when flight first evolved and what changes this entailed, and what the patterns of diversification of birds have been since the evolution of flight. Similar questions can be asked about each branch of the tree of life.

As Moran (1993) noted, some details of life's history are insufficiently established to warrant designation as "facts," but this can (and probably will) change as more data are brought to bear on particular issues. For example, it is now an accepted fact that dinosaurs were the dominant terrestrial vertebrates for a period of 160 million years and that they disappeared comparatively abruptly about 65 million years ago. It is not yet clear, however, what the implications of this mass extinction event were for the subsequent evolution of the mammals and birds who now fill many of the niches previously occupied by dinosaurs (BinindaEmonds et al. 2007; Wible et al. 2007).

As with its status as scientific fact and its nature as theory, there are often confusions about the path of evolution among nonspecialists. The erroneous notion that humans descended from chimpanzees or monkeys falls into this category. Chimps and humans are not related as ancestors and descendants, but rather as cousins whose lineages last shared a common ancestor about 6 million years ago. A great deal of change has occurred along both lineages since their split from this common ancestor, and many species have come and gone along both lines of descent. 


\section{Debates About Theory and Path do not Impinge on Fact}

Evolutionary biology has as its purview the entire history and diversity of life, encompassing an unbroken chain of ancestry and descent involving innumerable organisms and spanning billions of years. In light of the tremendous scope and complexity of its subject matter, it should come as no surprise that details regarding the path and mechanisms of evolution are often subject to heated debate. The fact of evolution, however, remains unsinged. To quote Gould (1981) once again,

Scientists regard debates on fundamental issues of theory as a sign of intellectual health and a source of excitement. Science is - and how else can I say it?most fun when it plays with interesting ideas, examines their implications, and recognizes that old information might be explained in surprisingly new ways. Evolutionary theory is now enjoying this uncommon vigor. Yet amidst all this turmoil no biologist has been led to doubt the fact that evolution occurred; we are debating how it happened.

Is evolution always gradual, or can it follow a more punctuated pattern? Are chance mechanisms such as genetic drift ever as important as the nonrandom process of natural selection? Does natural selection operate only among organisms (or genes) within populations, or can it occur at other levels such as among groups or species? Did mammals diversify as a consequence of the extinction of dinosaurs? Is the primary divide among groups of organisms between those with and those without nuclei, or are there deeper splits? Are wholescale genome duplications common in evolution, and if so, are they associated with major evolutionary changes? Can complex features ever be regained once they have been lost from a lineage? Is a substantial fraction of noncoding DNA functional, or is most of it simply "junk" or "parasitic"? Was Australopithecus afarensis ("Lucy") a direct ancestor of Homo sapiens or a member of a different hominid lineage? Debate over these questions of theory and path can become quite acrimonious within evolutionary biology, but in no case do they raise doubt about the fact of evolution. As Gould (1981) noted, "facts do not go away when scientists debate rival theories to explain them."

In broader terms, evidence for a given fact can be accepted at the same time that a proposed explanation for it is rejectedthis is, after all, precisely what happened in Darwin's case as mutationism, orthogenesis, and neo-Lamarckism competed with natural selection before the rise of the Modern Synthesis. Responding to critics in 1871, Darwin wrote:

Some of those who admit the principle of evolution, but reject natural selection, seem to forget, when criticising my book, that I had the above two objects in view; hence if I have erred in giving to natural selection great power, which I am very far from admitting, or in having exaggerated its power, which is in itself probable, I have at least, as I hope, done good service in aiding to overthrow the dogma of separate creations.

Indeed, Darwin was very explicit about the distinction between descent with modification (fact) and natural selection (theory). As he noted in 1863:

Whether the naturalist believes in the views given by Lamarck, or Geoffroy St.-Hilaire, by the author of the 'Vestiges,' by Mr. Wallace and myself, or in any other such view, signifies extremely little in comparison with the admission that species have descended from other species and have not been created immutable; for he who admits this as a great truth has a wide field opened to him for further inquiry.

Unfortunately, conflation of fact and theory in this regard is not limited to opponents of evolution. Some biologists make the inverse mistake of considering clear evidence of common descent as evidence that it occurred by natural selection. Certainly, one can propose that natural selection is responsible for any changes that show evidence of having been adaptive, but change through time (evolution as fact or path) does not, by itself, evince any particular mechanism (evolution as theory). Neither this failure to distinguish between fact or path and theory by scientists, nor that perpetuated by antievolutionists, is compatible with a proper understanding of the scientific definitions of these terms.

\section{Concluding Remarks}

It has been noted many times that evolution is both a fact and a theory (Gould 1981; Moran 1993; Futuyma 1998; Lenski 2000). It can also be considered in terms of a historical path (Ruse 1997). The fact of evolution, that organisms alive today are related by descent from common ancestors, is fundamental to an understanding of biology. As Dobzhansky (1973) famously stated, "nothing in biology makes sense except in the light of evolution". Nevertheless, a great deal remains to be determined regarding the mechanisms that have created (and destroyed) biological diversity since the emergence of life on Earth. Put in another way, modern evolutionary biology rests upon an extraordinarily solid foundation supported by multiple pillars of evidence, while its theoretical framework remains under construction. That the edifice of evolutionary theory is not yet complete is no cause for concern. Indeed, this is what makes evolutionary biology such an exciting and dynamic modern science. 


\section{References}

Alters BJ, Nelson CE. Teaching evolution in higher education. Evolution 2002;56:1891-901.

Bennett AW. The theory of natural selection from a mathematical point of view. Nature 1870;3:30-33.

Bininda-Emonds ORP, Cardillo M, Jones KE, MacPhee RDE, Beck $\mathrm{RMD}$, Grenyer R, et al. The delayed rise of present-day mammals. Nature 2007:446:507-12.

Bishop BA, Anderson CW. Student conceptions of natural selection and its role in evolution. J Res Sci Teach 1990;27:415-27.

Bowler PJ. The eclipse of Darwinism. Baltimore, MD: Johns Hopkins University Press; 1992.

Bowler PJ. Evolution: the history of an idea. 3rd ed. Berkeley, CA: University of California Press; 2003.

Darwin CR. Origin of species [Letter]. Athenaeum 9 May: 617; 1863.

Darwin C. On the origin of species by means of natural selection, or the preservation of favoured races in the struggle for life. London: John Murray; 1859.

Darwin C. The descent of man, and selection in relation to sex. London: John Murray; 1871.

Demastes SS, Settlage J, Good R. Students' conceptions of natural selection and its role in evolution: cases of replication and comparison. J Res Sci Teach 1995;32:535-50.

Dobzhansky T. Nothing in biology makes sense except in the light of evolution. Am Biol Teach 1973;35:125-9.
Futuyma DJ. Evolutionary biology. 3rd ed. Sunderland, MA: Sinauer Associates; 1998.

Gould SJ. Evolution as fact and theory. Discover 1981 May:34-37.

Halloun IA, Hestenes D. The initial knowledge state of college physics students. Am J Phys 1985a;53:1043-55.

Halloun IA, Hestenes D. Common sense concepts about motion. Am J Phys 1985b;53:1056-65.

Kampourakis K, Zogza V. Students' preconceptions about evolution: how accurate is the characterization as "Lamarckian" when considering the history of evolutionary thought? Sci Educ 2007;16:393-422.

Kramer M, Stairs IH, Manchester RN, McLaughlin MA, Lyne AG, Ferdman RD, et al. Tests of general relativity from timing of the double pulsar. Science 2006;314:97-102.

Lenski R. Evolution: fact and theory. ActionBioScience.org http:/ www.actionbioscience.org/evolution/lenski.html; 2000.

Mayr E, Provine WB. The evolutionary synthesis. Cambridge, MA: Harvard University Press; 1980.

Moran L. Evolution is a fact and a theory. Talk.Origins http://www. talkorigins.org/faqs/evolution_fact.html; 1993.

National Academy of Sciences of the USA. Teaching about evolution and the nature of science. Washington, DC: National Academies Press; 1998.

Randall L. Warped passages. New York: Harper Perennial; 2005.

Ruse M. Taking Darwin seriously. Amherst, NY: Prometheus Books; 1997.

Wible JR, Rougier GW, Novacek MJ, Asher RJ. Cretaceous eutherians and Laurasian origin for placental mammals near the K/T boundary. Nature 2007;447:1003-6. 